\title{
Dinámica de la gestión de la innovación de servicios y co-creación en empresas del sector economía digital
}

\section{Dynamics of service innovation management and co-creation in firms in the digital economy sector}

\author{
Rafael Luis Herrera González ${ }^{1 *}$, Antonio Hidalgo Nuchera² \\ ${ }^{1}$ Universidad de Costa Rica, Costa Rica \\ ${ }^{2}$ Universidad Politécnica de Madrid, España
}

Recibido el 14 de septiembre de 2017; aceptado el 17 de octubre de 2018

Disponible en Internet el: 30 de noviembre de 2018

\section{Resumen}

En este trabajo se analiza el proceso de la gestión de innovación en el sector TIC que se enmarca en el contexto de la economía digital, prestando especial énfasis en las actividades de co-creación que realizan las empresas. Tomando como punto de partida las principales consideraciones teóricas sobre la innovación en servicios se plantea un modelo de innovación de seis fases basado en el modelo TEMAGUIDE, al cual se le incorporan dos nuevas fases, una relativa a la gestión de los recursos humanos y otra relativa a la capacidad de co-creación. Los resultados obtenidos ponen de relieve que la agregación de valor requiere de realizar procesos de co-creación en donde se involucren diferentes actores.

Código JEL: M11, M30, O30, O31, Q33

Palabras clave: Innovación; servicios; gestión de la innovación; co-creación; economía digital; TIC.

\footnotetext{
* Autor para correspondencia

Correo electrónico: rafael.herrera@ucr.ac.cr (R. L. Herrera González)

La revisión por pares es responsabilidad de la Universidad Nacional Autónoma de México.
} 


\begin{abstract}
This paper analyzes the process of innovation management in the ICT sector that is framed in the context of the digital economy, with special emphasis on the co-creation activities carried out by companies. Taking as a starting point the main theoretical considerations on innovation in services, a six-phase innovation model based on the TEMAGUIDE model is proposed, which incorporates two new phases, one related to the management of human resources and the other relative to the capacity of co-creation. The results obtained show that the aggregation of value requires to carry out co-creation processes where different actors are involved.
\end{abstract}

JEL code: $\mathrm{M} 11, \mathrm{M} 30, \mathrm{O} 30, \mathrm{O} 31, \mathrm{Q} 33$

Keywords: services; innovation management; co-creation; digital economy; ICT.

\title{
Introducción
}

De acuerdo con Tapscott (1995), la economía digital se caracteriza por tres aspectos fundamentales: se basa en la aplicación de los conocimientos humanos a todo lo que se produce y cómo se produce, lo que permite afirmar que cada vez más valor añadido se creará por el cerebro en lugar de la fuerza muscular; está ayudando a modificar la estructura del actual sistema económico a través de la convergencia entre la informática (hardware, software), las comunicaciones (telefonía por cable, satélite, inalámbrica), y contenidos (entretenimiento, editoriales, proveedores de información); y la información en todas sus formas se convierte en digital y se reduce a bits almacenados en computadoras que se distribuyen a gran velocidad a través de las redes. Como consecuencia de ello, el número de negocios que se generan en el sector de la economía digital muestra un crecimiento exponencial (Elkelsen, Marcus y Ferree, 2009), dentro de las cuatro formas de clasificar las empresas que configuran el sector de economía digital: bienes y servicios digitales puros; bienes y servicios digitales mixtos; producción de bienes y servicios intensivos en TIC; e industria de las TIC (Fundación OEI, 2010).

La revolución de las tecnologías de información y comunicación (TIC) ha permitido que ésta se convierta en una herramienta indispensable para la puesta en práctica de numerosos procesos de transformación socioeconómica (Castells, 2001), en particular la denominada economía digital. Las TIC han ayudado a mejorar el funcionamiento en sectores de servicios antes estancados al facilitar la comunicación, reducir los costes de transacción, permitir la interconexión y la cooperación entre empresas, así como acelerar los procesos de innovación (OECD, 2000). Es en esta perspectiva donde algunos estudios se enfocan en analizar como las TIC contribuyen, impactan o son un medio para generar innovaciones en sectores de servicios como el financiero, la educación, la salud o el desarrollo empresarial (Añón, 2011; Ollo-López y Aramendía-Muneta, 2012; Lechman y Marszk, 2015; Andreassen, Kjekshus, y Tjora, 2015; Pavela, Fruthb y Neacsu, 2015). En otros casos se ha estudiado el rol de las TIC para el uso y eficacia de los procesos de innovación Kroh, Luetjen, Globocnik, and Schultz, (2018). Por su parte, Yunis, El-Kassar y Tarhini, (2017), analizan el impacto de las innovaciones basadas en las TIC sobre el rendimiento de la organización. 
El sector servicios se ha convertido en uno de los principales creadores de riqueza y su crecimiento ha sido muy relevante en las últimas décadas, alcanzando en 2014 el $71 \%$ del Producto Interno Bruto a nivel mundial. En las economías más avanzadas, los servicios generan hasta tres cuartas partes de la riqueza y el $85 \%$ del empleo, lo que hace que cada vez sea mayor la demanda por entender los elementos claves de la gestión de la innovación en este tipo de organizaciones (Tidd y Bessant, 2009; Goffin y Mitchell, 2010). En Costa Rica, el Banco Central reveló que el sector servicios pasó de representar un $10 \%$ de la producción del país en 1966, a un $28 \%$ en 1991 y a un $40 \%$ en 2012, convirtiéndose en una de las actividades más importantes.

En el caso de las TIC, este sector representó el 5.5\% del valor añadido total de los países de la OCDE en 2013, lo que equivale a alrededor de 2.4 billones de dólares. Los productos informáticos, electrónicos y ópticos, y la edición de software han representado el $1.4 \%$ y el $0.3 \%$ del valor añadido total, respectivamente, generando un total de 2.6 millones de empleos (OECD, 2015). Asimismo, entre 1995 y 2014, las exportaciones mundiales de servicios de informática e información aumentaron a un ritmo muy superior al de los demás sectores de servicios, registrando una tasa media anual de crecimiento del 18\%. Según el Banco Central de Costa Rica (2018), para el 2016 Costa Rica realizó exportaciones de servicios por medio de TIC por un valor de USD 3.310 millones, equivalente al 5.8\% del Producto Interno Bruto (PIB). Ese porcentaje es similar a lo que genera la actividad agrícola en el país. Del total del valor exportado, el $88 \%$ corresponde a compañías de gran tamaño, aunque también hay una participación importante de microempresas $(7 \%)$. El restante $5 \%$ se reparte entre la mediana y pequeña empresa. En cuanto al destino de las exportaciones, el $60 \%$ se dirige a los Estados Unidos de América, $13 \%$ a Europa, y $12 \%$ a Centroamérica.

Estos datos ponen de manifiesto que las empresas del sector TIC han tenido un impulso muy relevante en las últimas décadas. Esta industria, se caracteriza por tener una dinámica altamente cambiante, el ambiente en que se desenvuelven, impulsado por el acelerado avance de la tecnología, del conocimiento, y los requerimientos de los clientes, las obliga a buscar modelos para la agregación de valor, esto hace que sea esencial conocer cómo las empresas innovan y como gestionan procesos. Hay estudios que analizan como se lleva a cabo la innovación en el sector TIC, Lee, C., Hyun, J. and Lee, D., 2017, y en algunos de sus subsectores que lo componen, por ejemplo en software Yigitcanlar, T. et al., (2018) y en la innovación de servicios empresariales basada en la nube Ali, Warren, and Mathiassen, (2017).

Los servicios empresariales intensivos en conocimiento (KIBS), se consideran una piedra angular de las economías desarrolladas contemporáneas, Mustak, M. (2017). En el caso específico de las empresas de servicios del sector TIC, éstas se enmarcan en lo que se denomina empresas intensivas en conocimiento (KIBS, por sus siglas en inglés) Figueiredo, Vieira, Gonçalves, y de Matos, (2017). En términos generales, este tipo de empresas dependen en gran medida de los conocimientos profesionales, pues utilizan el conocimiento para producir servicios intermedios para los procesos de producción de sus clientes (Torrosi, 1998).

El objetivo de este estudio es analizar el proceso de gestión de innovación en las empresas del sector TIC, que se enmarcan en el contexto de economía digital, prestando especial énfasis en las actividades de co-creación. El artículo está organizado de la siguiente manera: la sección 2 muestra una revisión de las consideraciones teóricas sobre la innovación en los servicios y la co-creación; la sección 3 presenta la propuesta de un modelo que permite analizar la gestión de la innovación a través de diferentes fases; y la sección 4 describe los principales resultados 
del estudio. Finalmente, en la sección 5 se muestran las principales conclusiones y limitaciones del estudio.

\section{Revisión de la literatura}

El sector servicios es una parte muy diversificada de la economía que abarca desde sectores intensivos en tecnología y conocimientos, como servicios informáticos o de negocios, hasta servicios poco tecnológicos y poco cualificados como la mayor parte de los servicios personales (COTEC, 2004). El servicio suele ser interactivo, con un elevado nivel de participación y de contacto entre los suministradores de servicios y los clientes en el diseño, la producción, el suministro, el consumo y otras fases de la actividad (Miles, 2005). Es un conjunto de actividades y actos de comunicación, enlazados a través de secuencias parciales o totales, que son realizadas de forma simultánea por el cliente, el servidor y sus proveedores, para alterar el estado del cliente, o de un objeto de su interés o propiedad, con un fin, en un entorno cultural determinado (D’Alvano e Hidalgo, 2011). Diferentes autores hacen referencia sobre las principales características de los servicios: intangibilidad, heterogeneidad e inseparabilidad (Lovelock, 1983; Parasuraman, Zeithaml y Berry, 1985; Kotler, 1997; Miles, 2008; Tidd y Bessant, 2009; Goffin y Mitchell, 2010).

\section{La innovación en servicios}

Se define el concepto de innovación como "la introducción de un nuevo producto (bien o servicio), o de uno significativamente mejorado, un proceso reciente, un método novedoso de comercialización, o un moderno sistema organizativo en las prácticas internas de una empresa, la organización del lugar de trabajo o se aplica en las relaciones exteriores" (OCDE, 2005). Desde la perspectiva de Hidalgo et al. (2002), la innovación es un proceso dinámico de la utilización eficiente de la base de conocimientos de la organización para desarrollar productos nuevos o mejorados de manera diferente. Por su parte, la innovación en el sector de servicios comprende nuevos servicios y nuevas formas de producción o prestación de servicios, así como cambios significativos en los servicios, en su producción y en la forma en que se entrega. De acuerdo con Den Hertog et. al. (2010) es "una nueva experiencia de servicio o solución de servicio, que puede consistir en un nuevo servicio, una nueva cartera de servicios y/o un nuevo proceso de servicio que de forma individual o en combinación, define una nueva forma de crear valor para el cliente".

A pesar de la importancia de la innovación del servicio, el concepto está en constante evolución, en este sentido, Witell et al. (2016), analizan y caracterizan la innovación en servicios, de acuerdo con tres diferentes perspectivas: asimilación; demarcación; y síntesis. Asimismo, Snyder et al. (2016), sobre la base de una revisión extensa y sistemática de 1046 artículos académicos, exploraron cómo definen y utilizan los investigadores la innovación del servicio. A tal efecto, identifican cuatro categorías únicas de innovación de servicios que enfatizan los siguientes rasgos: (1) grado de cambio, (2) tipo de cambio, (3) novedad y (4) medios de provisión.

Tradicionalmente la gestión de la innovación y los modelos para su análisis han estado suscritos a la innovación tecnológica y a la industria manufacturera (Gallouj y Weisten, 
1997; Chesbrough, 2011). Para Tidd y Bessant (2009) la mayoría simplemente asume que tales prácticas son igualmente aplicables a la gestión de la innovación en los servicios, pero algunos investigadores argumentan que los servicios son fundamentalmente diferentes. Sin embargo, la creciente importancia que los servicios van teniendo en los índices globales de crecimiento ha generado que en la actualidad este sea uno de los temas prioritarios de estudio entre diferentes disciplinas relacionadas con la innovación (Miles, 2005; Tidd y Bessant, 2009; Goffin y Mitchell, 2010). Estos dos últimos autores consideran que las propias características de los servicios tienen una implicación directa en la gestión de la innovación, mientras que Chesbrough (2011) plantea que el aumento de los servicios en esta nueva etapa provoca que estos enfoques deban cambiar si las empresas quieren ser exitosas y sostenibles en el tiempo.

\section{El proceso de innovación y la co-creación}

El proceso de innovación en servicios implica un alto grado de interactividad entre el proveedor de servicios y el cliente (Zeithaml y Bitner, 2003). Desde la perspectiva de Markovic, and Bagherzadeh, (2018), la co-creación puede generar una multitud de ventajas organizacionales, incluido un mejor rendimiento de la innovación. La participación de diferentes actores en la co-creación de servicios y en los procesos de innovación ha sido referenciada por autores como Chesbrough (2003 y 2011) que hace énfasis en la importancia de los agentes externos para la innovación en sus planteamientos sobre innovación abierta. Otros autores hacen referencia a la co-creación para la agregación de valor, la innovación y participación de los clientes (Prahalad y Ramaswamy, 2004; Vargo y Lusch, 2008; Tanev et al., 2010; Mele, Russo y Colurcio, 2010; Grönroos, 2011; Mukhat, Nazul y Yahya, 2012; Von Bischhoffshausen, Hottum y Straub, 2015, Raeisi y Lingjie, 2017, Cui, y Wu, 2017, AnningDorson, 2018, Mahmoud, Hinson y Anim, 2018, Cui y Wu, 2018).

En particular, Greer, Lusch y Vargo (2016) plantean cinco axiomas que caracterizan esta teoría: en primer lugar, el servicio es la base fundamental del intercambio; en segundo lugar, el valor es co-creado por múltiples actores, siempre incluyendo el beneficiario; en tercer lugar, todos los actores sociales y económicos son integradores de recursos; en cuarto lugar, el valor siempre está determinado de forma única y fenomenológica por el beneficiario; y, en quinto lugar, la co-creación se coordina a través de instituciones y arreglos institucionales generados por actores. Por último, en el tema de creación de valor y su relación con la co-creación se pueden distinguir diferentes enfoques, incluyendo la lógica S-D, la ciencia de servicios (Maglio y Spohrer, 2008), la lógica de servicio, el marketing many-to-many, el construccionismo social, el desarrollo de nuevos productos o el posmodernismo (Saarijärvi y Hannu, 2013). Vargo, S., Lusch, R. (2016 y 2017), han incorporado una extensión o actualización de la teoría de la lógica dominante del servicio, y plantean que esta puede continuar avanzando durante la próxima década, hacia un mayor desarrollo de una teoría general del mercado y, aún más ampliamente, hacia una teoría general de la co-creación de valor. 


\section{Aspectos metodológicos}

\section{Propuesta de modelo}

Para analizar cómo las empresas de servicios TIC gestionan sus procesos de innovación se propone un modelo basado en el modelo TEMAGUIDE elaborado por la Fundación COTEC y que incorpora una serie de módulos con sus respectivas herramientas que las empresas pueden utilizar en la gestión del proceso de innovación (modelo GI_TIC). Aunque este modelo fue concebido inicialmente para la industria manufacturera, posteriormente se han realizado varias aproximaciones para utilizarlo en el sector servicios (COTEC, 2004; D’Alvano e Hidalgo, 2011). De este modelo se mantienen las áreas vigilar, aprender e implantar, y se agrupan los aspectos de estrategia en la etapa de focalizar.

Con la finalidad de identificar las singularidades que presentan las empresas del sector servicios TIC se incorporan al modelo dos nuevas áreas: recursos humanos y co-creación. El área de recursos humanos, se parte de los planteamientos de Arzola y Mejías (2007), se recoge la importancia del conocimiento en este sector y la necesidad de incorporar mecanismos de capacitación. El área de co-creación trata de tener en cuenta la perspectiva en la cual los diferentes actores (clientes, proveedores, universidades) pueden participar activamente en los procesos de creación de valor y de innovación. Especial interés tiene el análisis de aspectos como las estrategias de desarrollo de proyectos y la participación de los actores en diferentes etapas (ideación, desarrollo de producto e implementación), el rol de los clientes en el proceso de co-creación (Von Bischhoffshausen, Hottum y Straub, 2015), y las técnicas utilizadas para realizar actividades de co-creación (Mukhat, Nazul y Yahya, 2012). La figura 1 representa el modelo propuesto.

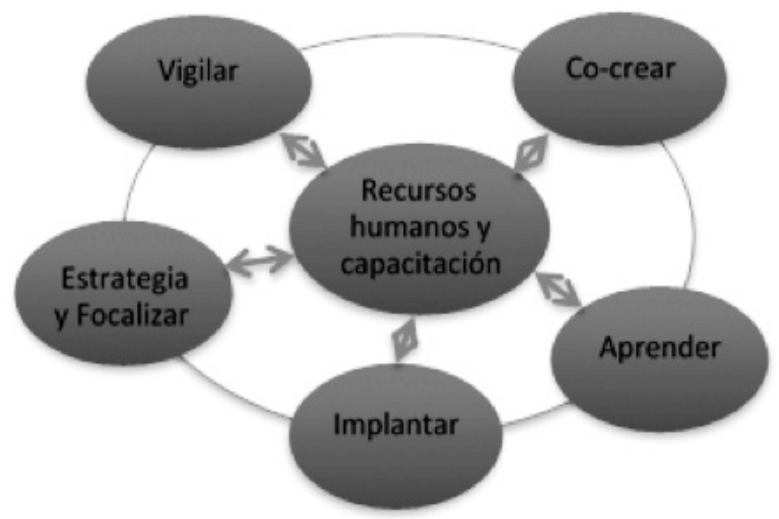

Figura 1. Modelo de gestión de la innovación en servicios TIC.

\section{Definición de la muestra y variables}

Para la validación del modelo GI_TIC se elaboró un cuestionario basado en estudios contrastados. Para las etapas recursos humanos, vigilar, focalizar, implantar y aprender, se 
consideró el modelo TEMAGUIDE (COTEC, 2004) y el cuestionario utilizado por D’Alvano e Hidalgo (2012), pero aplicado al sector TIC. Para la fase de co-creación y la relativa a estrategia de proyectos se utilizaron las aportaciones de Pikkarainen et al.(2011) y Von Bischhoffshausen, Hottum y Straub (2015). El cuestionario fue estructurado en siete módulos, con un total de 37 preguntas, el primero de ellos sobre información general de la empresa (7), y los restantes seis se corresponden con las fases del modelo: Recursos Humanos (6); Co-creación (6); Vigilar (4); Estrategia y Focalizar (6); Implantar (5) y Aprender (3).

La encuesta fue aplicada a gerentes de empresas del sector TIC en Costa Rica entre los meses de junio y agosto de 2016. La población total a estudiar inicialmente estaba constituida por 170 empresas asociadas a la Cámara de Tecnologías de Información y Comunicación en Costa Rica (CAMTIC), aunque después de una preselección se consolidó un total de 140 empresas de los subsectores tecnologías de información, comercialización de tecnologías, desarrollo de software, e-commerce, multimedia digital, servicios habilitados de tecnología digital, y juegos.

El cuestionario fue enviado utilizando una plataforma electrónica (e-encuesta), y se obtuvo respuesta de 47 empresas (33.6\% del total), clasificadas de la siguiente manera: 19 del subsector desarrollo de software (40.4\%), 17 del subsector de tecnologías de información (36.2\%), 4 del subsector e-commerce (8.5\%), 2 del subsector de comercialización de tecnologías (4.3\%) y 5 del subsector considerado como otros que incluye videojuegos, realidad aumentada y virtual, regulación de TIC, seguridad informática (10.6\%). Los datos fueron procesados en el SPSS (version 19) y algunos gráficos en programa R versión 3.3.1. Se determinó el alpha de Cronbach's que obtuvo un valor de 0.877 , lo que pone de manifiesto la existencia de una adecuada consistencia interna entre las variables.

\section{Índice de gestión de la innovación en servicios TIC}

Con la finalidad de analizar la capacidad de innovación de las empresas estudiadas se definió el índice de gestión de la innovación en servicios TIC (IGIS_TIC), que es un indicador del proceso de innovación que realizan las empresas según el modelo planteado. Este indicador varía de 0 a 100 promediando el puntaje en el subíndice de cada fase. Se obtiene del promedio simple de los subíndices de cada una de las fases del modelo: recursos humanos, co-creación, vigilancia, estrategia y focalizar, implantar y aprender (Tabla 1).

Para la construcción de los índices hay que tener en cuenta la existencia de tres niveles: las variables (20), los subíndices o fases (6) y el índice general (1). Cada variable o pregunta según su formato y cantidad de categorías de respuesta se promedian en un solo indicador. En la mayoría de los casos, las preguntas utilizadas solicitaban respuestas de si o no para una serie de técnicas, acciones o aspectos relevantes, de ahí que se sumen todas y se promedien para obtener un solo valor para esa pregunta, la cual se estandariza en una escala de 0 a 100 . Una vez definidas todas las variables, se genera el subíndice conformado por el promedio de los indicadores de todas las preguntas. Para cada una de las seis fases del modelo de innovación se utilizaron una serie de variables para determinar cómo se gestiona el proceso de innovación. 
Tabla 1

Variables y definición de subíndices.

\begin{tabular}{|c|c|c|c|}
\hline INDICE & SIGLA & VARIABLES UTILIZADAS & DEFINICIÓN \\
\hline $\begin{array}{l}\text { RECURSOS } \\
\text { HUMANOS }\end{array}$ & I_DRH & $\begin{array}{l}\text { Actividades de adquisición del conocimiento (9) } \\
\text { Herramientas de gestión de innovación ha capacitado } \\
\text { al personal (8) } \\
\text { Relevancia recurso humano para innovación (5) }\end{array}$ & $\begin{array}{l}\text { Se obtuvo un índice simple } \\
\text { que promedia } 3 \text { variables } \\
\text { relacionadas con el papel del } \\
\text { Recurso Humano para las } \\
\text { empresas. }\end{array}$ \\
\hline $\mathrm{CO}-\mathrm{C}$ & IC_OC & $\begin{array}{l}\text { Etapas del proyecto, según estrategia y actores co- } \\
\text { crean en el desarrollo del proyecto ( } 9 \text { y } 3 \text { actores) } \\
\text { Técnicas para realizar actividades de co-creación, } \\
\text { según actores (10) }\end{array}$ & $\begin{array}{l}\text { Se calculó un índice (simple } \\
\text { o no ponderado) de } 2 \text { variables } \\
\text { relacionadas con la Co-creación } \\
\text { que realizan las empresas según } \\
\text { las estrategias utilizadas y los } \\
\text { actores. }\end{array}$ \\
\hline VIGILAR & I_V & $\begin{array}{l}\text { Actividades de vigilancia (8) } \\
\text { Actividades de fuentes de información (9) } \\
\text { Herramientas vigilancia (10) }\end{array}$ & $\begin{array}{l}\text { Se calculó un índice (simple o } \\
\text { no ponderado) de } 3 \text { variables de } \\
\text { la etapa Vigilar. }\end{array}$ \\
\hline
\end{tabular}

FOCALIZAR I_F Estrategia e innovación (3)

Actividades I+D+i últimos 3 años (8)

Participación actores co-creación innovación (6)

Se calculó un índice (simple o

Proceso selección ideas (6) no ponderado) de 6 variables de

Actividades generación ideas (6)

la etapa Focalizar.

Herramientas para la selección ideas (9)

\begin{tabular}{llll}
\hline IMPLANTAR & I_I & Estrategia desarrollo proyectos (4) & \\
& & Técnicas planeación proyectos innovación (9) & $\begin{array}{l}\text { Se calculó un índice (simple o } \\
\text { no ponderado) de 4 variables de } \\
\text { la etapa Implantar. }\end{array}$ \\
& & $\begin{array}{l}\text { Herramientas para implantar innovación (10) } \\
\text { Razones para desarrollo de proyectos innovación }\end{array}$ & \\
\hline APRENDER & I_A & Actividades aprendizaje de la empresa (4) & $\begin{array}{l}\text { Se calculó un índice (simple o } \\
\text { no ponderado) de 2 variables de } \\
\text { la etapa Aprender". }\end{array}$ \\
\hline
\end{tabular}

Nota: el contenido en (), columna 3, refiere al número de acciones o ítems utilizados en cada variable de análisis. Fuente: Elaboración propia con base en Encuesta de Gestión de Innovación el sector TIC. Costa Rica, 2016.

Después de crear los subíndices se obtiene el índice IGIS_TIC con el promedio simple de ellos (recursos humanos, co-crear, vigilar, focalizar, implantar y aprender), cuya fórmula es:

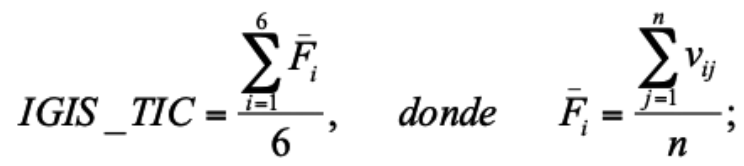




\section{Correlaciones entre índice general y subíndices}

En la figura 2 se presenta la matriz de correlaciones entre el índice de gestión de innovación en servicios TIC (IGIS_TIC) con los subíndices que componen el modelo. Los resultados muestran que todas las correlaciones son significativas con respecto al IGIS_TIC, en todos los casos con un valor mayor a 0.7 . Las relaciones son directas y positivas, lo que significa que al aumentar el valor de un determinado índice aumenta el otro, lo que permite indicar y justificar el uso de los índices para el desarrollo del IGIS_TIC. Además, es importante indicar que entre los subíndices también hay relaciones positivas.

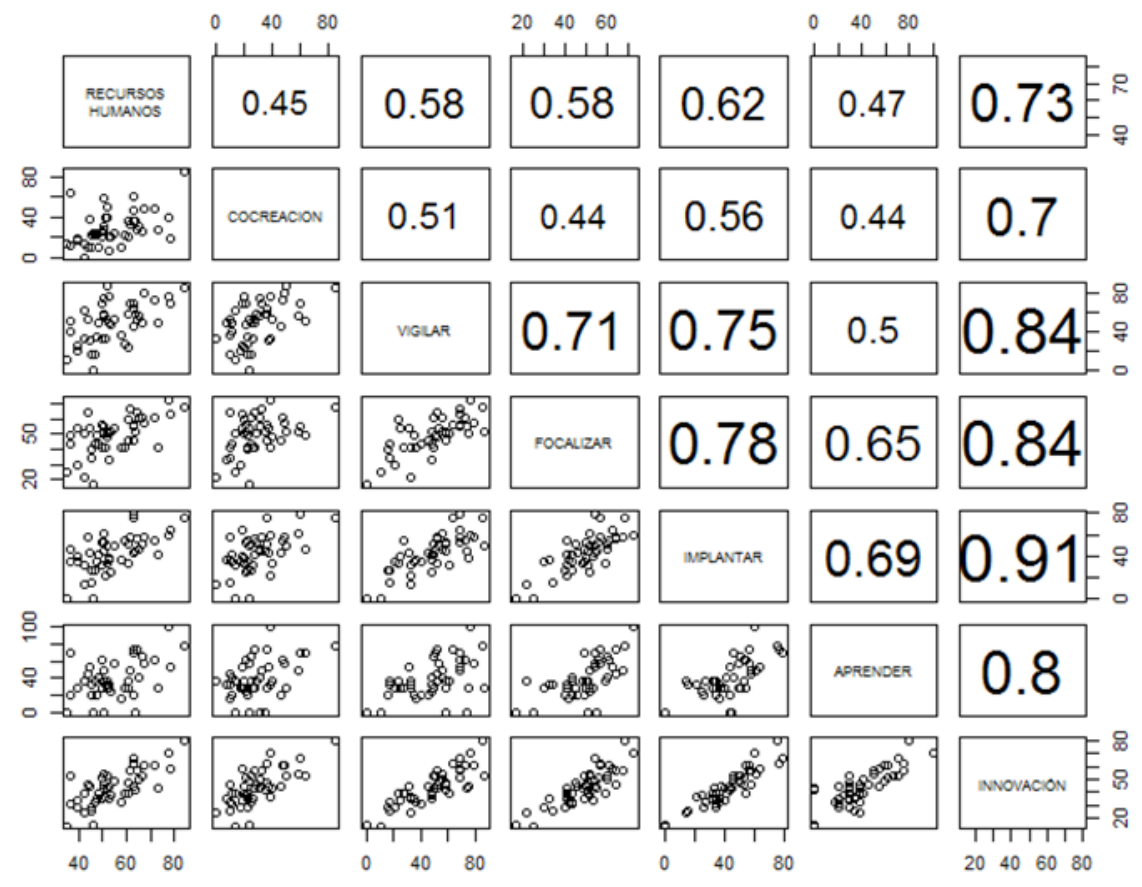

Figura 2. Matriz de correlaciones del modelo

Fuente: Elaboración propia con base en Encuesta de Gestión de Innovación el sector TIC, Costa Rica. 2016.

Índices co-crear, clientes, socios-proveedores y universidades

El índice co-crear (I_COC) se obtiene del promedio simple de dos variables, una de las cuales resulta de la combinación de nueve posibles opciones de co-crear para cada actor, derivado de las tres estrategias y tres etapas de ejecución del proyecto. También se consideró para el cálculo la suma de diez técnicas de co-creación que utilizan las empresas. La definición matemática del índice es:

$$
I_{-} C O C=\frac{\sum_{i=1}^{m} v(t)_{i}}{m}
$$


Adicionalmente se generan tres subíndices: clientes (I-CL), socios-proveedores (I_SP) y universidades (I-UNIV), que se calculan de igual forma que el de co-crear, con la diferencia que de que en estos casos cada subíndice se refiere a los actores que se seleccionaron para este estudio (cliente, socios y proveedores, universidades).

Con el objetivo de analizar la significatividad de estos subíndices se elaboró una regresión múltiple con relación al índice de innovación (variable dependiente). Como resultado se obtuvo que todas las variables analizadas dan significativas al 0,1 , siendo el valor del ajuste $\mathrm{R}^{2}$ de 0.449 y el estadístico Durbin-Watson de 1.939.

\begin{tabular}{|c|c|c|c|c|c|}
\hline \multirow{2}{*}{$\begin{array}{l}\text { Modelo } \\
\text { B }\end{array}$} & \multicolumn{2}{|c|}{$\begin{array}{l}\text { Coeficientes no } \\
\text { estandarizados }\end{array}$} & \multirow[t]{2}{*}{$\begin{array}{c}\text { Coeficientes } \\
\text { tipificados }\end{array}$} & \multirow{2}{*}{$\mathrm{t}$} & \multirow{2}{*}{ Sig. } \\
\hline & Error típ. & Beta & & & \\
\hline (Constante) & 27.102 & 3.365 & & 8.055 & .000 \\
\hline INDICE_CLIENTE & .215 & .065 & .384 & 3.289 & .002 \\
\hline INDICE_SOCIOS & .176 & .049 & .410 & 3.592 & .001 \\
\hline INDICE_UNIVERSIDADES & .183 & .103 & .205 & 1.770 & .084 \\
\hline
\end{tabular}

a. Variable dependiente: INDICE_INNOVACION

En la figura 3 se presenta la matriz de correlaciones entre el IGIS_TIC con los subíndices I_COC, I_CL, I_SP, I_UNIV. Los resultados muestran que todas las correlaciones son significativas, que hay relaciones directas y positivas con respecto al I_COC, que los subíndices de clientes y proveedores tiene un valor igual o mayor a 0.7 , mientras que en el caso de las universidades el subíndice es menor (0.5). No obstante, los subíndices con respecto al IGIS_TIC son positivos, aunque sus valores son menores a 0.5. Es importante señalar que las relaciones entre los actores son muy bajas, lo que no es significativo en el análisis, ya que lo que se estudia es la relación de la empresa con los actores y no como interactúan ellos entre sí.

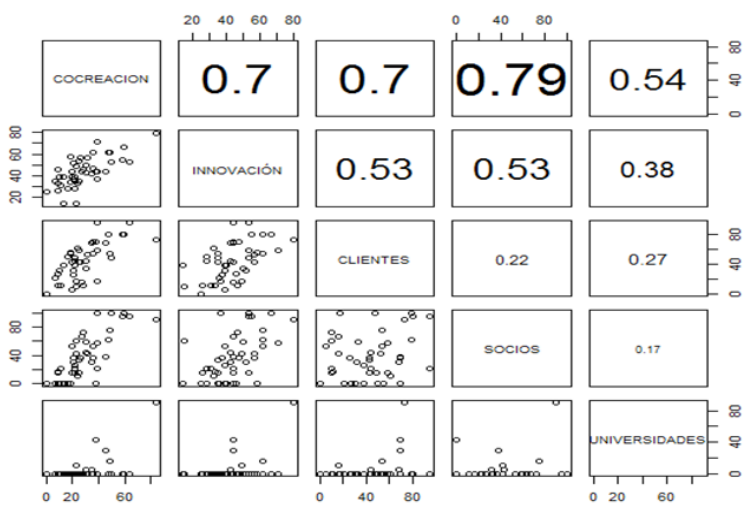

Figura 3. Matriz de correlaciones entre co-crear y gestión de innovación

Fuente: Elaboración propia con base en Encuesta de Gestión de Innovación el sector TIC, Costa Rica. 2016. 


\section{Características de las empresas}

De acuerdo con Pavitt (1984), los sectores tienen diferentes prioridades y características que condicionan a las empresas por los efectos de la innovación, las economías de escala y la intensidad científica. Respecto al tamaño, Rothwell (1985) señalan que éste es un factor que tiene especial repercusión en cuanto al acceso a los recursos y otros factores. El uso de estas variables es reforzado por Tidd y Bessant (2009), quienes agregan que estos aspectos pueden afectar la gestión de la innovación de las empresas.

La figura 4, recoge el valor promedio del índice IGIS_TIC y de los subíndices que representan la capacidad de co-creación. Se observa que cuando las empresas tienen un menor número de empleados, es decir, entre 1 y 30, el valor promedio del índice general de gestión innovación está por debajo del promedio. Esta situación es inversa cuando las empresas tienen más de 30 empleados, lo que permite concluir que a mayor tamaño de la empresa mejora su índice de gestión de innovación. Este panorama cambia cuando se analizan los subíndices relacionados con la co-creación, dado que, con excepción de las empresas de 11 a 30 empleados, en el subíndice de clientes, el valor de la media es mayor al promedio, y solo las empresas grandes (con más de 100 empleados) tienen valores medios superiores en los subíndices con respecto al promedio general. Como complemento a lo anterior, después de hacer una regresión múltiple, tomando como variable dependiente el IGIS_TIC, y las variables "subsector" y "número de empleados", los datos indican que solo el número de empleados es significativo, es decir, que por cada empleado que tenga una empresa el IGIS_TIC aumenta en 0.363 puntos.

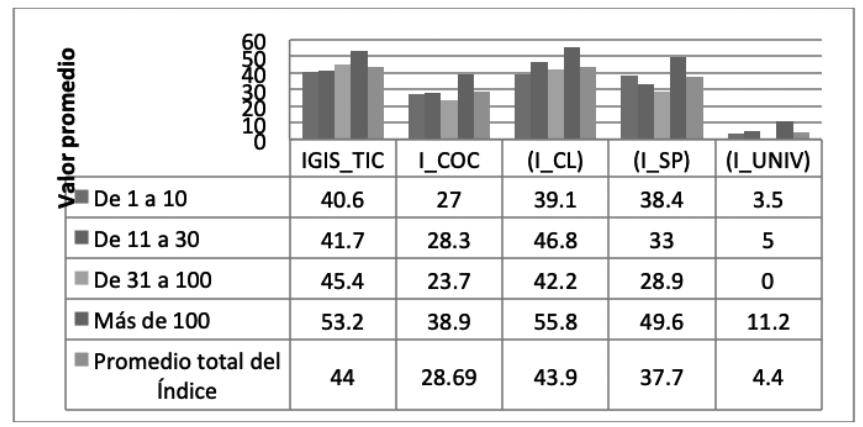

Figura 4. Valor promedio de los índices (IGIS_TIC y I_COC) y los subíndices (I_CL, I_SP y I_UNIV), según tamaño de la empresa

Fuente: Elaboración propia con base en Encuesta de Gestión de Innovación el sector TIC, Costa Rica. 2016.

\section{Subsector}

Al analizar el subsector al que pertenece la empresa, los resultados permiten identificar que, cuando las empresas pertenecen a los subsectores de desarrollo de software y comercialización de tecnologías, tienen valores promedios inferiores al promedio general del subíndice respectivo. Cuando la co-creación se da con los clientes, el respectivo subíndice es mayor que el valor medio para las empresas de tecnologías de información y de e-commerce, que cuando se da con los proveedores y las universidades. Aunque en algunos subsectores los subíndices 
de co-creación y actores están por debajo de la media, esta situación es más favorable cuando se analiza el índice general de innovación IGIS_TIC. En este caso solo las empresas de comercialización de tecnología presentan un valor inferior al promedio general (Figura 5).

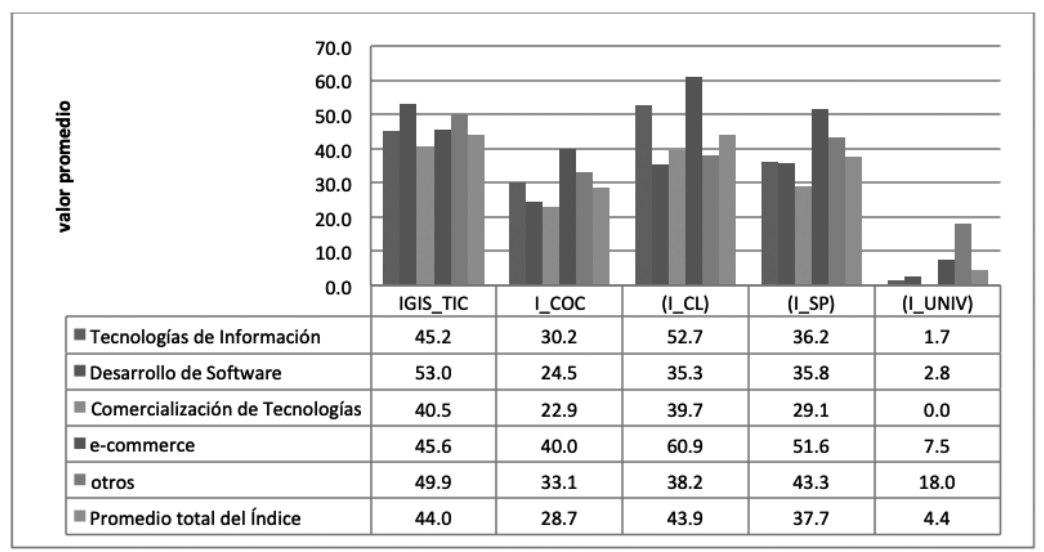

Figura 5. Valor promedio de los índices (IGIS_TIC y I_COC) y los subíndices (I_CL, I_SP y I_UNIV), según subsector.

Fuente: Elaboración propia con base en Encuesta de Gestión de Innovación el sector TIC, Costa Rica. 2016.

\section{Actores y su relación con el tipo de estrategia}

$\mathrm{Al}$ analizar cómo se comportan los índices relacionados con co-creación y actores, y su relación con el tipo de estrategia para el desarrollo de proyectos, las empresas participantes en el estudio que utilizan cada una de las estrategias, en la gran mayoría de los casos los índices tienen valores más altos al promedio (Tabla 2). Se destaca que los que usan estrategia tienen mayores valores en cada subíndice con respecto a los que no la usan, lo que indica que las empresas que implementan estas estrategias tienen procesos de innovación más eficientes.

Actores y su participación en las etapas del proyecto

El análisis de la participación de los actores en las etapas del desarrollo del proyecto (ideación, desarrollo del proyecto, implementación y adaptación) pone de relieve que en las empresas que utilizan la estrategia "basada en proyectos" se da una participación de los clientes en la etapa de ideación y en la de implementación. Esta situación varía cuando se colabora con socios y proveedores, donde su mayor participación se produce en la etapa de desarrollo de producto. Este comportamiento es el mismo que cuando el actor es la universidad (Figura 6).

En las empresas que utilizan la estrategia "out-the-box" se da una participación mayor de los clientes en la etapa de ideación y en la de implementación. Cuando la colaboración se produce con los socios y proveedores hay mayor participación, y en el mismo porcentaje, en las etapas de ideación y de desarrollo de producto. Para el actor universidad la mayor participación se da en la etapa de desarrollo. 
En aquellas empresas que utilizan la estrategia "desarrollo de productos a la medida" se da una participación mayor de los clientes en la etapa de ideación y en la de implementación. Cuando la colaboración es con los socios-proveedores y con la universidad, hay mayor participación en la etapa de desarrollo de producto.

Tabla 2

Valor promedio de los subíndices de co-crear y actores según estrategia.

\begin{tabular}{lcccc}
\hline ESTRATEGIA/INDICE & I_COC & I-CL & I-SP & I-UNIV \\
\hline Estrategia de desarrollo basada en proyectos & & & & \\
NO & 12.4 & 20.1 & 16.9 & 0.0 \\
SI & 32.7 & 49.8 & 42.9 & 5.5 \\
Estrategia Out-of-the-box, de desarrollo de productos & & & & \\
NO & 19.3 & 34.3 & 23.7 & 0.0 \\
SI & 33.8 & 49.1 & 45.4 & 6.8 \\
Estrategia de desarrollo de productos a la medida & & & & \\
NO & 20.3 & 31.1 & 28.2 & 1.7 \\
SI & 31.7 & 48.5 & 41.1 & 5.4 \\
TOTAL & 28.69 & 43.9 & 37.7 & 4.4 \\
\hline
\end{tabular}

Fuente: Elaboración propia con base en Encuesta de Gestión de Innovación el sector TIC, Costa Rica. 2016.

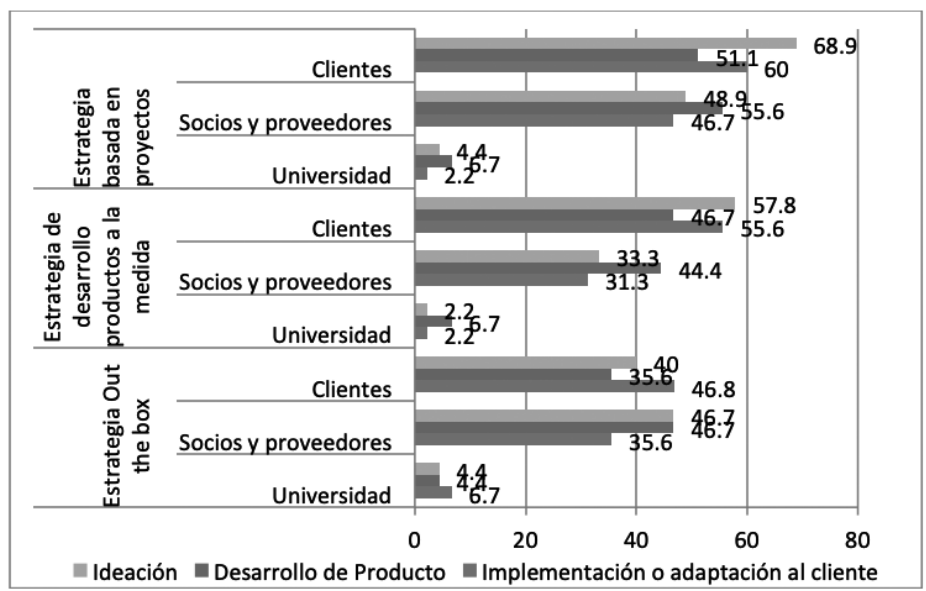

Figura 6. Participación de los actores en la etapa de desarrollo de proyectos según estrategia.

Fuente: Elaboración propia con base en Encuesta de Gestión de Innovación el sector TIC, Costa Rica. 2016. 


\section{El rol del cliente en la co-creación}

De acuerdo con la perspectiva planteada por Von Bischhoffshausen, Hottum y Straub (2015), los clientes pueden tener diferentes roles en el proceso de co-creación. Es de interés conocer los valores que se obtienen al analizar los roles de los clientes con respecto a los índices de innovación, co-creación y el subíndice cliente. Como se puede observar en la tabla 3 , para todos los roles de los clientes el índice de innovación es mayor al promedio. Por otra parte, cuando el cliente actúa como especificador del servicio y como auditor de calidad, el índice de co-creación es menor al promedio. Para el subíndice cliente, cuando el cliente actúa como auditor de calidad, éste disminuye en relación al promedio. El hecho de que cuando se usa una determinada función o técnica es mayor al promedio se debe a que tiene un valor más alto de quienes no las usan.

Tabla 3

Valor promedio del índice co-crear y el subíndice cliente según el rol del cliente.

\begin{tabular}{|c|c|c|c|c|c|c|}
\hline \multirow[t]{2}{*}{ Rol del cliente } & \multicolumn{2}{|c|}{ I_COC } & \multicolumn{2}{|c|}{ I_CL } & \multicolumn{2}{|c|}{ IGIS_TIC } \\
\hline & SI & NO & SI & NO & SI & NO \\
\hline $\begin{array}{l}\text { Co-diseñador: ayuda como "consultor" durante el proceso de diseño } \\
\text { y toma de decisiones. }\end{array}$ & 33.6 & 23.4 & 49.8 & 37.7 & 46.5 & 41.3 \\
\hline $\begin{array}{l}\text { Especificador de servicio: proyecta el servicio de entrega a través de } \\
\text { su especificación. }\end{array}$ & 27.0 & 32.0 & 45.8 & 40.0 & 44.4 & 43.0 \\
\hline $\begin{array}{l}\text { Co-mercadólogo: apoya la mercadotecnia del servicio a través de } \\
\text { publicidad de boca en boca. }\end{array}$ & 32.0 & 28.0 & 49.5 & 42.8 & 51.9 & 42.5 \\
\hline $\begin{array}{l}\text { Auditor de calidad: asegura la calidad de la producción y entrega a } \\
\text { través de pruebas abiertas y quejas. }\end{array}$ & 28.3 & 29.0 & 43.3 & 44.5 & 45.4 & 42.3 \\
\hline $\begin{array}{l}\text { Co-productor: Provee el insumo en forma de factores de la producción: } \\
\text { trabajo, conocimiento, información. }\end{array}$ & 38.1 & 22.3 & 55.2 & 36.3 & 48.4 & 41.0 \\
\hline TOTAL & 28.6 & & 43.9 & & 44.0 & \\
\hline
\end{tabular}

Fuente: Elaboración propia con base en Encuesta de Gestión de Innovación el sector TIC, Costa Rica. 2016.

\section{Principales resultados}

Definidas las variables del estudio, el índice IGIS_TIC y los respectivos subíndices y sus correlaciones, el análisis se centró en identificar como se lleva a cabo el proceso de cocreación dentro de la gestión de la innovación utilizando las variables "números de empleados" y "subsector" al que pertenecen las empresas. En relación con el tema de co-crear, se analiza especialmente la participación de los actores de acuerdo con la estrategia de la empresa y las diferentes etapas del proyecto, el rol del cliente y las herramientas más utilizadas en las etapas de co-crear.

\section{Uso de técnicas y herramientas}

El uso de técnicas y herramientas de gestión son relevantes en los estudios relacionados con la innovación y ha sido considerado un factor importante para el éxito de la gestión de 
la innovación (Hidalgo y Albors, 2008; Igartua, Albors y Hervas, 2010; Teza et al., 2016, Harrington, y Voehl, 2016, Albors, Igartua, y Peiro, 2018). Por su parte, D’Alvano e Hidalgo (2012) aplican las técnicas de gestión de innovación para estudiar el grado de desarrollo del proceso de innovación en las organizaciones de servicios (comercio, salud y educación). Albors, J., Igartua, J. y Peiro, A. (2018), analizan cómo la utilización de las Técnicas de Gestión de la Innovación (IMTS) influye en el rendimiento de la innovación de las empresas. En el ámbito de las herramientas relacionadas con innovación y co-creación, Füller et al. (2009) estudian cómo es el empoderamiento del consumidor a través de la co-creación basada en Internet, por su parte, Pallot et al. (2010) estudian el uso de Living Lab en ambientes de investigación, desde el diseño centrado en el usuario y su experiencia en la co-creación, mientras que Kohler et al. (2011) analizan las consecuencias de la experiencia de co-creación virtual y el uso de avatares como oportunidad para que las empresas aprovechen el potencial innovador de los consumidores y las comunidades de consumidores.

En el estudio se consultó a las empresas sobre qué herramientas son más o menos usadas, de acuerdo con el tipo de actor con que se relacionan en los procesos de innovación y cocreación. Como se muestra en la figura 7, las herramientas utilizadas no son homogéneas para los tres actores y el grado de uso varía de acuerdo al tipo de actor. No obstante, las herramientas de métodos participativos (focus group, buzones de ideas y diseño empático) obtienen los porcentajes mayores de uso con independencia del tipo de actor. Cuando las empresas co-crean con los clientes, la herramienta co-diseño y uso de artefactos y prototipos es relevante $(51,1 \%)$, mientras que con el actor proveedores es importante el uso de medios digitales $(46,7 \%)$. En el caso de la universidad, los métodos de innovación basados en comunidades de usuarios están entre los más usados. Por el contrario, las herramientas como "Living Lab", y "personas y avatares" no son muy utilizadas. El uso de medios digitales tiene a ser mayor cuando se trabaja con los socios y proveedores.

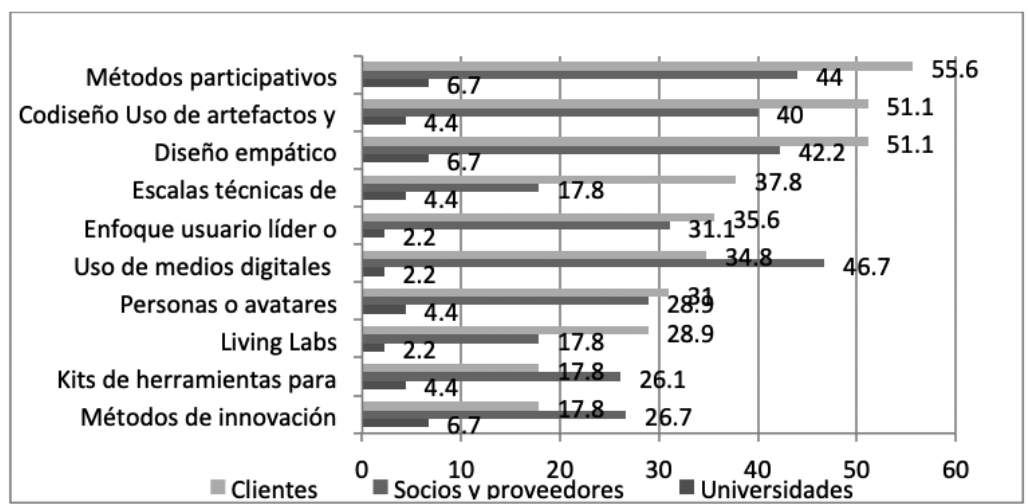

Figura 7. Porcentaje del uso de herramientas de co-creación según actor.

Fuente: Elaboración propia con base en Encuesta de Gestión de Innovación el sector TIC, Costa Rica. 2016. 


\section{Conclusiones}

En el ámbito económico, el sector servicios desde hace varias décadas ha tenido un crecimiento exponencial convirtiéndose en algunos países en la principal fuente de ingresos de la economía. Por su parte, la revolución de las TIC ha ocasionado una transformación productiva, y las redes interconectadas, la globalización del conocimiento y de los negocios han ampliado el acceso de los clientes y los usuarios a los bienes y servicios. Asimismo, los actores ya no son simplemente compradores, sino que se convierten en fuente y generadores de ideas para la innovación, y pueden participar en diferentes etapas de los proyectos.

Estos hechos han permitido un crecimiento importante de las empresas del sector TIC, las cuales juegan un rol relevante desde dos puntos de vista: como generadoras de bienes y servicios, y como fuente para que otros sectores de la economía aceleren sus procesos de innovación y mejoren los servicios que prestan a los consumidores. Desde esta perspectiva, las empresas TIC se enfrentan al reto de incrementar su capacidad para diferenciarse y agregar valor por medio de la innovación y, por ende, la implementación de sus procesos de gestión, a fin de garantizar a los clientes servicios de alta calidad y acordes con las necesidades del usuario. La agregación de valor requiere de realizar procesos de co-creación en donde se involucren diferentes actores.

Como resultado de este estudio se pueden mencionar los siguientes aspectos como de especial relevancia:

-El modelo propuesto de gestión de innovación en empresas de servicios de TIC se adecuan de manera significativa a empresas de servicios.

-El tamaño de la empresa sigue siendo un factor importante para que las empresas aumenten su capacidad de gestión de la innovación, es decir, a mayor tamaño el índice de gestión de innovación y los subíndices relacionados con la co-creación tienden a incrementarse. El tamaño también influye en la participación de los diferentes actores, por ejemplo, cuando las empresas son más grandes co-crean más con las universidades.

-Aunque se asume que los clientes son los principales actores en el proceso de co-creación, los resultados del estudio muestran que los socios y proveedores juegan un rol significativo en este proceso. Sin embargo, hay que destacar el escaso nivel de participación de las universidades en el proceso de co-creación. Una de las posibles razones, es que las empresas del sector TIC, por ser intensivas en conocimiento, al tener profesionales muy calificados y por el tipo de servicios que prestan, pueden realizar sus procesos de innovación a lo interno o con el apoyo de los clientes y los proveedores. Sin embargo, en este aspecto, es importante realizar más investigación, y ver si este comportamiento se da de igual manera en otros sectores menos incentivos en conocimiento.

- La participación de los actores en las diferentes etapas del desarrollo del proyecto varía de acuerdo con el tipo de estrategia que utilizan y el tipo de actor. A modo de ejemplo, en la estrategia basada en proyectos, los clientes participan más activamente en las etapas de ideación e implementación, aunque en el caso de los socios y proveedores tienen una mayor participación en la etapa de desarrollo.

-El uso de herramientas para co-creación varía dependiendo del tipo de actor, es decir, no se da homogeneidad en el tipo de herramienta que es usada por cada tipo de actor. 
En un contex to de intensificación de los procesos de globalización, donde se incrementan las exigencias de competitividad, una respuesta para generar mayor valor agregado a los productos y servicios dirigidos tanto al mercado externo como al local, es incrementar las actividades relacionadas con la gestión de la innovación y el trabajo conjunto con diferentes actores en las empresas de servicios de TIC, a fin de mejorar sus procesos de innovación y de agregación de valor, especialmente en las pequeñas y medianas empresas.

\section{Referencias}

Albors, J., Igartua, J. y Peiro, A. (2018). Innovation management techniques and tools: its impact on firm innovation perfomance. International Journal of Innovation Management, 22(6). https://doi.org/10.1142/s1363919618500512.

Ali, A., Warren, D. and Mathiassen, Lars (2017). Cloud-based business services innovation: A risk management model. International Journal of Information Management. 37 (6), 639-649. https://doi.org/10.1016/j.ijinfomgt.2017.05.008.

Andreassen, H., Kjekshus, L., Tjora, A. (2015). Survival of the project: A case study of ICT innovation in health care. Social Science \& Medicine, 132, 62-69. https://doi.org/10.1016/j.socscimed.2015.03.016

Anning-Dorson, Thomas (2018). Customer involvement capability and service firm performance: The mediating role of innovation. Journal of Business Research, 86, 269-280. https://doi.org/10.1016/j.jbusres.2017.07.015

Añón, D. (2011). The impact of ICT on innovation activities: Evidence for UK SMEs, International Small Business Journal 30(6), 684-699. https://doi.org/10.1177/0266242610374484

Arzola, M., Mejías A. (2007). Modelo conceptual para gestionar la innovación en empresas del sector servicios. Revista Venezolana de Gerencia 12(37), 80-98. Disponible en: http://www.redalyc.org/articulo.oa?id=29003706. Consultado: 14/10/2016

Banco Central de Costa Rica (2018). Resultados del Estudio: Exportaciones de servicios por medio de las Redes TIC. Mayo, 2018. https://activos.bccr.fi.cr/sitios/bccr/ComunicadosPrensa/Docs_Comunicados_Prensa/ICT_Project_CR_ESP.pdf

Castells, M. (2001). La Era de la Información. Siglo XXI Editores. México.

Chesbrough, H. (2003). Open innovation: the new imperative for creating and profiting from technology. Harvard Business School Press. Boston, Mass.

Chesbrough, H. (2011). Open Services Innovation. Rethinking Your Business to Grow and Compete in a New Era. HB Printing. EE.UU.

COTEC (2004). Análisis del proceso de innovación en las empresas de servicios. Madrid, España.

Cui, A. and Wu. F. (2017). The Impact of Customer Involvement on New Product Development: Contingent and Substitutive Effects. Journal of Product Innovation Management, 34, 1, (60-80). https://doi.org/10.1111/jpim.12326

Cui, A. and Wu, F. (2018). Customer Involvement in Innovation: A Review of Literature and Future Research Directions, in Rajan Varadarajan, Satish Jayachandran (ed.) Innovation and Strategy (Review of Marketing Research, Volume 15) Emerald Publishing Limited, pp.63 - 98. https://doi.org/10.1108/s1548-643520180000015005

D’Alvano, L., Hidalgo, A., (2011). Procesos de innovación en las organizaciones de salud y educación en Venezuela. Revista Venezolana de Gerencia 16(56), 578-594. Disponible en: http://www.redalyc.org/articulo. oa?id=29020563005. Consultado: 17/10/2016

D’Alvano, L., Hidalgo, A. (2012). Innovation management techniques and development degree of innovation process in service organizations. $R \& D$ Management 42(1), 60-70. https://doi.org/10.1111/j.1467-9310.2011.00663.x

Den Hertog, P., der Aa, W., de Jong, M. (2010). Capabilities for managing service innovation: towards a conceptual framework. Journal of Service Management 21(4), 490-514. ttps://doi.org/10.1108/09564231011066123

Elkelsen, G., Marcus, A., Ferree, W.K. (2009). The digital economy fact book 2008-2009. The Progress \& Freedom Foundation. Washington D.C. Disponible en: http://www.pff.org/digital-economy-fact-book/. Consultado: $14 / 9 / 2016$ 
Figueiredo, R., Vieira, J., Gonçalves, O. y De Matos, J. (2017). Knowledge Intensive Business Services (KIBS): bibliometric analysis and their different behaviors in the scientific literature Topic 16 - Innovation and services. RAI Revista de Administração e Inovação 14 (2017) 216-225. https://doi.org/10.1016/j.rai.2017.05.004

Füller, J., Mühlbacher, H., Matzler, K., Jawecki, G. (2009). Consumer empowerment through internet-based co-creation. Journal of Management Information Systems 26(3), 71-102. https://doi.org/10.2753/mis0742-1222260303

Fundación EOI (2010). Economía Digital. Serie Sectores de la nueva Economía 20+20. Madrid, España. Disponible en: http://www.eoi.es/blogs/20digital/. Consultado: 14/9/2016

Gallouj, F., Weinstein, O. (1997). Innovation in services. Research Policy 26, 537-556. https://doi.org/10.1016/S00487333(97)00030-9

Goffin, K., Mitchell, R. (2010). Innovation Management. Strategy and implementation using the pentathlon framework. Palgrave Macmillan. Second edition. https://doi.org/10.1007/978-1-137-04752-6

Greer, C., Lusch, R., Vargo, S. (2016). A service perspective: Key managerial insights from service-dominant (S-D) logic. Organizational Dynamics 45, 28-38. https://doi.org/10.1016/j.orgdyn.2015.12.004

Grönroos, C. (2011). Value co-creation in service logic: A critical analysis. Marketing Theory 11(3), 279-301. https:// doi.org/10.1177/1470593111408177

Hidalgo, A., León, G., Pavón, J. (2002). La Gestión de la Innovación y la Tecnología en las Organizaciones. Pirámide. Madrid.

Hidalgo, A., Albors, J. (2008). Innovation management techniques and tools: A review from theory and practice. $R \& D$ Management, 38(2), 113-127. https://doi.org/10.1111/j.1467-9310.2008.00503.x

Igartua, J., Albors, J., Hervas-Oliver, J. (2010). How innovation management techniques support an open innovation strategy. Research-Technology Management 53(3). https://doi.org/10.1080/08956308.2010.11657630

Harrington, J. (Editor) and Voehl, F. (Editor), (2016). The Innovation Tools Handbook, Volume 3: Creative Tools, Methods, and Techniques that Every Innovator Must Know. ISBN-13: 978-1498760539, 1st Edition. https://doi. org/10.1201/9781315367682

Kohler, T., Fueller, J., Stieger, D., Matzler, K. (2011). Avatar-based innovation: Consequences of the virtual co-creation experience. Computers in Human Behavior 27, 160-168. https://doi.org/10.1109/hicss.2010.78

Kotler, P. (1997). Mercadotecnia. Prentice-Hall. México.

Kroh, J., Luetjen, H., Globocnik, D., and Schultz, C. (2018). Use and Efficacy of Information Technology in Innovation Processes: The Specific Role of Servitization. Journal of Product Innovation Management (in press). https://doi. org/10.1111/jpim.12445.

Lechman, E., Marszk, A. (2015). ICT technologies and financial innovations: The case of exchange traded funds in Brazil, Japan, Mexico, South Korea and the United States. Technological Forecasting \& Social Change 99, 355-376. https://doi.org/10.1016/j.techfore.2015.01.006

Lee, C., Hyun, J. and Lee, D., (2017). Intra-industry innovation, spillovers, and industry evolution: Evidence from the Korean ICT industry. Telematics and Informatics 34 (2017) 1503-1513. https://doi.org/10.1016/j.tele.2017.06.013

Lovelock, C. H. (1983). Classifying services to gain strategic marketing insights. Journal of Marketing 47, 9-20. Classifying services to gain strategic marketing insights. https://doi.org/10.2307/1251193

Mele, C., Russo, T., Colurcio, M. (2010). Co-creating value innovation through resource integration. International Journal of Quality and Service Sciences, 2(1), 60-78. https://doi.org/10.1108/17566691011026603

Maglio, P.P., Spohrer, J. (2008). Fundamentals of service science. Journal of the Academy of Marketing Science, 36(1), 18-20. https://doi.org/1007/s11747-007-0058-9

Mahmoud, M., Hinson, R., and Anim, P. (2018). "Service innovation and customer satisfaction: the role of customer value creation", European Journal of Innovation Management, 21 (3), 402-422, https://doi.org/10.1108/EJIM-092017-0117

Markovic, S. and Bagherzadeh, M. (2018). How does breadth of external stakeholder co-creation influence innovation performance?. Analyzing the mediating roles of knowledge sharing and product innovation. Journal of Business Research, 88, 173-186. https://doi.org/10.1016/j.jbusres.2018.03.028

Miles, I. (2005). Innovation in Services. In The Oxford Handbook of Innovation. Edited by Fagerberg, J., Mowery D. and Nelson, R. Oxford University Press. https://doi.org/ 10.1093/oxfordhb/9780199286805.003.0016 
Miles, I. (2008). Patterns of innovation in service industries. IBM Systems Journal, 47(1). https://doi.org/10.1147/ sj.471.0115

Mukhat, M. Nazul, M. y Yahya, Y. (2012). A hierarchical classification of co-creation models and techniques to aid in product or service design. Computers in Industry 63(4), 289-297. https://doi.org/10.1016/j.compind.2012.02.012

Mustak, M. (2017). Customer participation in knowledge intensive business services: Perceived value outcomes from a dyadic perspective. Industrial Marketing Management http://dx.doi.org/10.1016/j.indmarman.2017.09.017

OECD (2000). Science, Technology and Innovation in the New Economy. OECD Observer. Paris. Disponible en: http:// www.oecd.org/science/sci-tech/1918259.pdf. Consultado: 10/11/2016

OECD (2015). OECD Digital Economy Outlook 2015. Paris. Disponible en: http://www.oecd.org/internet/oecd-digital-economy-outlook-2015-9789264232440-en.htm. Consultado: 14/10/2016

Ollo-López, A., Aramendía-Muneta, E. (2012). ICT impact on competitiveness, innovation and environment. Telematics and Informatics 29(2), 204-210. https://doi.org/10.1016/j.tele.2011.08.002

Pallot, M., Trousse, B., Senach, B., Scapin, D. (2010). Living Lab Research Landscape: From User Centred Design and User Experience towards User Co-creation. First European Summer School "Living Labs". Paris. Disponible en: https://hal.inria.fr/inria-00612632/document. Consultado: 01/10/2016

Parasuraman, A., Zeithaml, V., Berry, L. (1985). A conceptual model of service quality and its implications for future research. Journal of Marketing 49, 41-50. https://doi.org/10.2307/1251430

Pavela, A., Fruthb, A., Neacsu, M. (2015). ICT and e-learning - catalysts for innovation and quality in higher education. Procedia Economics and Finance 23, 704-710. https://doi.org/10.1016/s2212-5671(15)00409-8

Pavitt, K. (1984). «Sectoral Patterns of Technological Change: Towards a Taxonomy and Theory».Research Policy, 13, 343-373. https://doi.org/10.1016/0048-7333(84)90018-0

Pikkarainen, M., Codenie, W., Boucart, N., Heredia Alvaro, J.A. (2011). The Art of Software Innovation. Eight Practice Areas to Inspire your Business. Springer. https://doi.org/10.1007/978-3-642-21049-5.

Prahalad, C.K., Ramaswamy, V. (2004). Co-creating unique value with customers. Strategy and Leadership 32(3), 4-9. https://doi.org/10.1108/10878570410699249.

Raeisi, S., Lingjie, M., (2017). The Importance of Customer Engagement and Service Innovation in Value Co-Creation. International Journal of Economics and Management Engineering. 11 (4). http://waset.org/pdf/books/?id=62816\&pageNumber=97. Consultado: 19/04/2018

Rothwell, R. (1985). Reindustrialization and technology: Towards a national policy framework. Science and Public Policy, 12 (3), 113-130, https://doi.org/10.1093/spp/12.3.113

Saarijärvi H., Hannu, K. (2013). Value co-creation: theoretical approaches and practical implications. European Business Review 25(1), 6-19. https://doi.org/10.1108/09555341311287718

Snyder, H. at el. (2016). Identifying categories of service innovation: A review and synthesis of the literature. Journal of Business Research 69 (2016) 2401-2408. https://doi.org/10.1016/j.jbusres.2016.01.009

Tanev, S., Thomsen, M., Zheng, M. (2010). Value co-creation: From an emerging paradigm to next innovation practices. Proceedings of the 3rd ISPIM Innovation Symposium. Quebec, Canada. 12-15 December 2010. Disponible en: http://findresearcher.sdu.dk/portal/files/47896977/ISPIM_20010_Paper_Quebec_Canada.pdf. Consultado: $01 / 10 / 2016$

Tapscott, D. (1995). The Digital Economy: Promise and Peril in the Age of Networked Intelligence. McGraw-Hill.

Teza, P., Buchele, G., De Souza, J., Dandolini, G. (2016). Analysis of quantitative empirical papers on diffusion and adoption of methods, techniques and tools for innovation. Revista de Administração e Inovação 13 (2). https://doi. org/10.1016/j.rai.2016.03.004

Tidd, J., Bessant, J. (2009). Managing Innovation. Integrating Technological, Market and Organizational Change. 4th Edition. John Wiley \& Sons, Ltd.

Torrosi, S. (1998). Industrial Organisation and Innovation. An International Study of the Software Industry. Edward Elgar, Cheltenham, UK.

Vargo, S. Maglio, P., Akaka, M. (2008). On value and value co-creation: A service systems and service logic perspective. European Management Journal 26, 145-152. https://doi.org/10.1016/j.emj.2008.04.003 
Vargo, S., Lusch, R. (2008). Service-dominant logic: Continuing the evolution. Journal of the Academy of Marketing Sciences 36,1-10. https://doi.org/10.1007/s11747-007-0069-6

Vargo, S., Lusch, R. (2016). Institutions and axioms: an extension and update of service-dominant logic. Journal of the Acad. Mark. Sci. (2016) 44:5-23. https://doi.org/10.1007/s11747-015-0456-3.

Vargo, S., Lusch, R. (2017). Service-dominat logic 2025. International Journal of Research in Marketing, 34 (1), 4667. https://doi.org/10.1016/j.ijresmar.2016.11.001

Von Bischhoffshausen, J., Hottum, P., Straub, T. (2015). Service co-creation. Part of the series Service Science: Research and Innovations in the Service Economy, pp 261-296. Springer International Publishing. https://doi. org/10.1007/978-3-319-23195-2_8

Witell, L. et al. (2016). Defining service innovation: A review and synthesis. Journal of Business Research. Volume 69, Issue 8, August 2016, Pages 2863-2872. https://doi.org/10.1016/j.jbusres.2015.12.055

Yigitcanlar, T. et al (2018). Impact of funding sources on innovation: evidence from Brazilian software companies. $R \& D$ Management (in press) https://doi.org/10.1111/radm.12323.

Yunis, M., El-Kassar, A. and Tarhini, A. (2017). "Impact of ICT-based innovations on organizational performance: The role of corporate entrepreneurship", Journal of Enterprise Information Management, 30 (1), 122-141, https:/doi. org/10.1108/JEIM-01-2016-0040

Zeithaml, V., Bitner, M. (2003). Service Marketing: Integrating Customer Focus across the Firm. McrGaw-Hill. New York. 